\title{
Pain estimation after coronary angiography based on vital signs by using artificial neural networks
}

\author{
Mohammad Amin Younessi Heravi ${ }^{1}$, Akram Gazerani ${ }^{2}$, Zakiehe Amini ${ }^{3}$, Parisa Salimi ${ }^{4}$, \\ Zahra Falahi ${ }^{5}$, Mohsen Yaghubi ${ }^{6}$ \\ Author affiliations: \\ 1. Department of Medical Physics and Radiology, North Khorasan University of Medical Sciences, Bojnurd, Iran. \\ 2. Department of Emergency Medicine, Torbat Heydaryeh University of Medical Sciences, Torbat Heydaryeh, Iran. \\ 3. MSc, Department of Nursing, Faculty of Nursing, North Khorasan University of Medical Sciences, Bojnurd, Iran. \\ 4. MSc Student of Neonatal Nursing, Faculty of Nursing and Midwifery, Shahid Beheshti University of Medical Sciences, Tehran, Iran. \\ 5. MSc Student of Pediatric Nursing, Faculty of Nursing and Midwifery, Tehran University of Medical Sciences, Tehran, Iran. \\ 6. MSc (Perfusion Technology), Department of Extra-Corporeal Circulation (ECC), Razavi Hospital, Imam Reza International University, \\ Mashhad, Iran
}

Correspondence: Akram Gazerani, Moalem Sq, Neyshabur City, Iran; Phone: +98-5143333964; E-mail: gazerania1@mums.ac.ir

\section{Abstract}

Background: Coronary angiography is gold standard method to diagnose coronary arteries diseases. The aim of this study was to estimate pain after coronary angiography based on vital signs for determining best position by using artificial neural networks ANN.

Methodology: This study used a database containing 86 subjects that refer to angiography center. For each subject Vital signs were measured that included blood pressure, percent of blood oxygen saturation, heart rate, respiratory rate and temperature. The Numeric Rating scale (NRS) was used to determine pain intensity. The vital signs were the inputs and the pain value was the corresponding output. These data were applied to train the ANN in the learning process. The model was implemented in MATLAB software. The results of pain estimation were compared with the results of NRS method and the error rate was calculated.

Results: The absolute error and error percentage between NRS method and the present method were $5.41 \pm 2.63 \mathrm{mmHg}, 4.09 \pm$ $1.59 \%$. The results indicated that the pain measurement by NRS method and pain value predicted with trained ANN differ by only less than $11 \%$. It is obvious that the neural network prediction fit properly to the NRS results.

Conclusion: The results of proposed method were closely in agreement with the results of the NRS. so this method can be suggested for reliving the pain and determining the best patient's position after the angiography procedure.

Key words: Artificial neural network; Coronary angiography; Pain

Citation: Heravi MAY, Gazerani A, Amini Z, Salimi P, Falahi Z, Yaghubi M. Pain estimation after coronary angiography based on vital signs by using artificial neural networks. Anaesth. pain intensive care 2021;25(1):29-34. DOI: 10.35975/apic.v25i1.1433.

Received: 21 November 2020, Reviewed: 2 December 2020, Accepted: 12 December 2020

\section{Introduction}

The coronary arteries supply the heart muscle with blood. They can become clogged from a buildup of cholesterol, cells or other substances (plaque). This can reduce the flow of blood to your heart. If a blood clot forms and blocks blood flow through that artery, a heart attack may occur. ${ }^{1}$ One of the most common and important diagnostic methods for detection of coronary arteries diseases are coronary angiography. This procedure is gold standard for CAD diagnosis. ${ }^{2}$ The coronary arteries supply oxygen-rich blood to heart so Coronary angiography is a test that shows the insides of coronary arteries. After angiography, vascular complications (such as; bleeding, hematoma and distal emboli due to trauma caused in artery wall), are most common complication of catheterization. One of the caring measures taken to reduce these complications is taking a complete or relative rest for 10 or 24 hours without moving legs. ${ }^{3}$ Although it is accepted by majority of specialists, unsuitable position and excessive immobility in bed after angiography, 
cause patients to experience pain in their low back. Lunden and colleagues conducted a qualitative study and assess the patient's responses after angiography and finally they realized that the pain is frequently reported following cardiac catheterization. ${ }^{4}$ The pain is an unpleasant sensory and emotional experience that created by annoying stimulation of sensory nerve

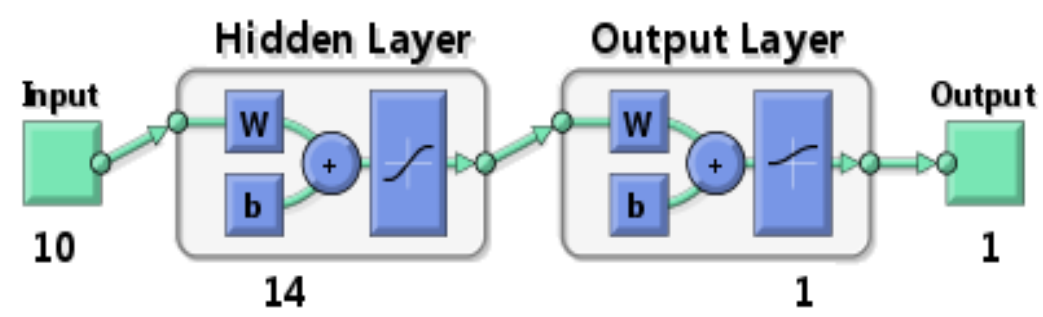
terminals. ${ }^{5}$ Some studies indicate that intramuscular pressure in lumbar muscles has direct relation to position of them. Rest in unchanged position for a long period of the time lead to increase tissue pressure and decrease blood supply via patient's bedridden. ${ }^{6}$ In parallel, activation of sympathetic system and catecholamine release due to pain cause to increase blood pressure, heart rate, respiratory rate, and decrease respiratory volume, and finally myocardial infarction..$^{7-10}$ Thus, despite the possibility of cardiovascular system involvement in patient who undergoing angiography, necessity of prevention and control of pain control in this patient is very important and accordingly there isn't any scientific support for a current method, ${ }^{11}$ Therefore, changing the exits treatment method after angiography (includes a complete rest on the back and unchanging position for a long period of time) and finding an optimal angle of bed to decrease patient's backache and their dissatisfaction, could be lead to faster improvement and also decrease their hospitalization. So, to relieve patient's pain without increasing the probable coronary complications during the complete bed rest, the best situation must be determined. Artificial neural network (ANN) is one of the artificial intelligence methods and it is finding many uses in the medical diagnosis application. ${ }^{12}$ Medical Diagnosis using Artificial Neural Networks is currently a very active research area in medicine and it is believed that it will be more widely used in biomedical systems in the next few years. ${ }^{13}$ ANNs attempt to find the desirable relation between the input and the corresponding output parameters during the learning process. A neural network learns by determining the relation between the inputs and outputs. The trained ANNs can predict the output of new unknown samples. ${ }^{14,15}$

The aim of this study was to develop ANN model for prediction the pain based on vital signs after coronary 
In this study, the vital signs were the inputs and the pain value was the corresponding output. These data were applied to train the networks in the learning process. The model was implemented in MATLAB software. The ANN toolbox of MATLAB provides a comprehensive set of functions and a graphical user interface for the design, implementation, visualization, and simulation of neural networks. ${ }^{17}$

For obtaining the desired results, the multilayer feed-forward back propagation was utilized. This network consists of three types of neuron layers that contain one input layer, one or several hidden layers and one output layer. Usually, for the training of this network the most popular learning algorithm called back propagation is applied. The back-propagation algorithm method is based on learning the ANN by adjusting the connection

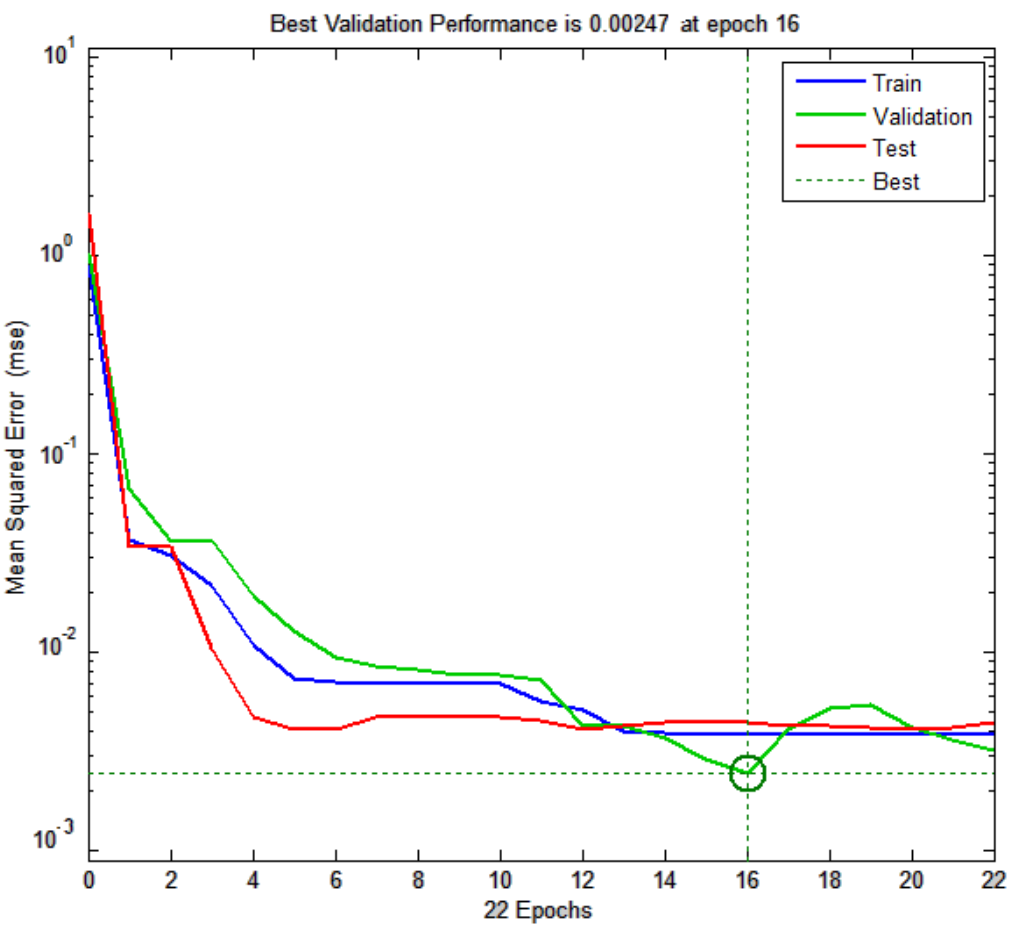

Figure 2: The circumstances of optimum network learning process

weights in order to minimize the network errors of prediction (error between the desired and real output values). ${ }^{14}$ The network training is an iterative process.

If the network error reduces to a minimum, the training process goes toward stability. Activation function determines the relationship between inputs and outputs

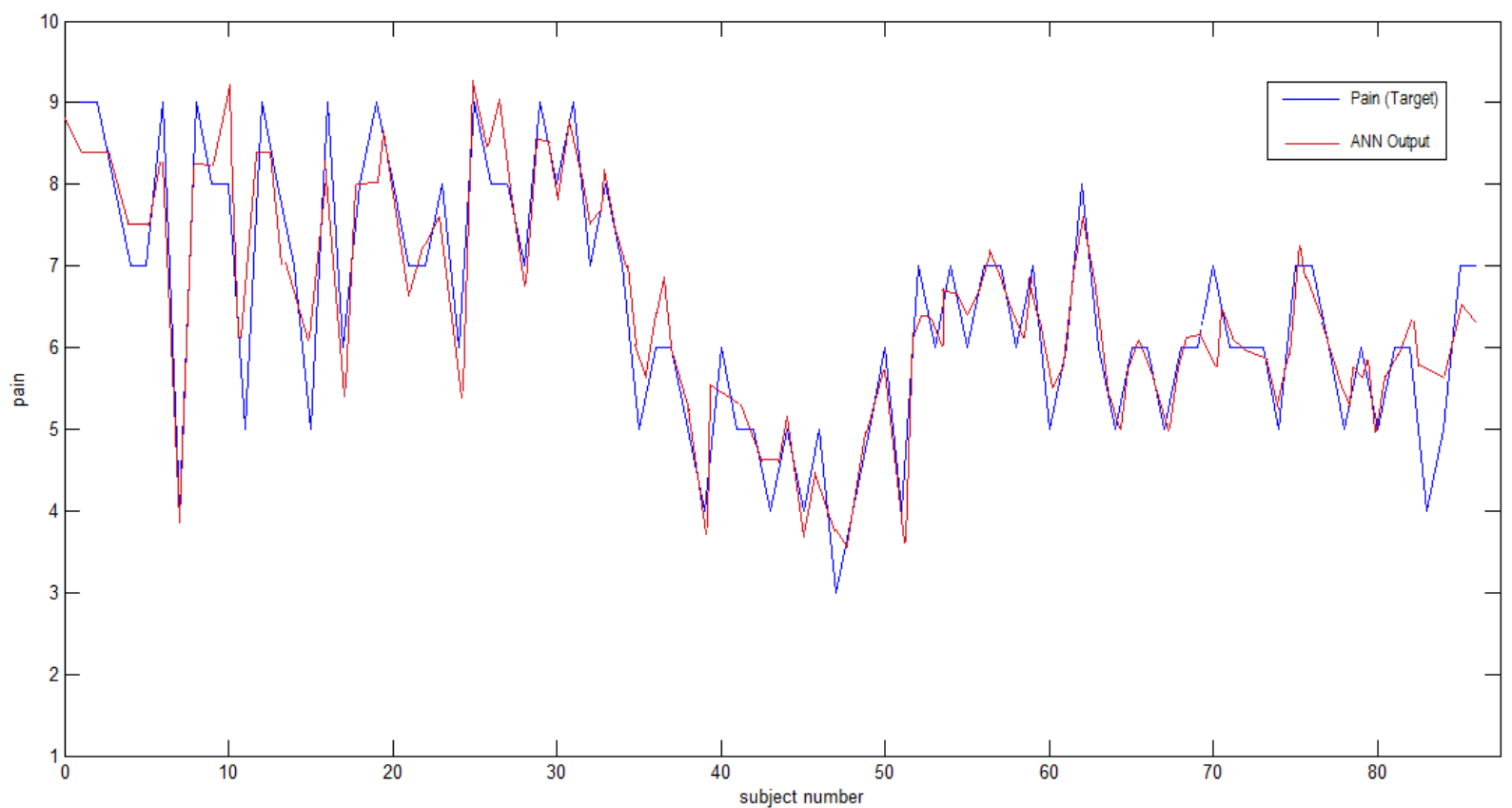

Figure 3: the prediction pain value (ANN output) and the pain from NRS method (target). 
of the nodes. There are different activation functions. The most popular ones such as LOGSIG and TANSIG were utilized to achieve the best performance.

The ability of the neural network model to predict the pain was evaluated by the mean square error (MSE) and coefficient of determination $\left(\mathrm{R}^{2}\right)$ between the measured and predicted data. The accuracy of neural network mode measured by the $\mathrm{R}^{2}$ parameter explains the model's goodness of fit. ${ }^{18,19}$ The presented data was randomly divided into training, validating and testing the network with appropriate architecture and learning algorithms. Training data are presented to the network during training and adjusting the connection weights to minimize the network errors of prediction. Validation data are utilized to assess network generalization. Testing data have no effect on training and give an independent measure of network performance during and after the training. In this study, the different activation function effects were examined on the network performance. For obtaining the optimum architecture of network, the numbers of layers and neurons in each layer were increased step by step.

\section{Results}

In this study, 86 patients participated, 37 (43\%) were men and 49 (57\%) were women.

The architecture of the optimal network model was feed forward with 10 input nodes, fourteen nodes in the hidden layer and an output node. The node in the output layer represented the pain value and the nodes in the input layer represented the systolic blood pressure, diastolic blood pressure, blood oxygen saturation, heart rate, respiratory rate, temperature, gender, age, height and weight. These inputs and corresponding output are applied as training patterns. Figure 1 illustrates the structure of the designed ANN.

Figure 2 illustrates the learning process for optimum network model by the present samples using MSE on all epochs.

The optimal network presented MSE $=2.47 \mathrm{E}-3$ and $\mathrm{R}^{2}$ $=0.801$ in 26 epochs with TANSIG and LOGSIG activation function for hidden and output layers respectively.

Figure 3 shows the pain which obtained from the ANN and the target pain. Absolute error and error percentage between NRS and trained ANN were $0.86 \pm 0.63$ and $\% 10.9 \pm 6.52$ maximum error was calculated 1.8 and
$26 \%$. By comparing these two sets of results, it has been found that the trained ANN could properly predict the value of the pain.

\section{Discussion}

This paper presents a new method to estimate the value of the pain for making suitable patients position after the angiography. The results showed that the absolute error and error percentage between NRS method and trained ANN were $0.86 \pm 0.63$ and $\% 10.9 \pm 6.52$. The results indicated that the pain measurement by NRS method and pain predicted with trained ANN differ by only less than $11 \%$. It is obvious that the neural network prediction could fit properly to the NRS results.

In Yaghubi's study was shown that, 45-degree angle was the best point of the bed to reduce pain after the procedure. The results of trained ANN showed that the pain value was dramatically reduced in 45 degree. The results of trained ANN were in a good arrangement with Yaghubi's study. This finding indicates that patient's recovery after coronary angiography in the flat position will cause severe pain experience.

In Various researches, the effect of changing the position was investigated as a pain reducing factor ${ }^{2}, 18$, ${ }^{19}, 20$ In these studies it was shown that changing the position caused a significant reduction in pain intensity at different times after the procedure, also no significant difference was seen in blood pressure and heart rate in the participants. Also, the study Adaryani and et $\mathrm{al}^{3}$ was shown that blood pressure and heart rate in the control group were dramatically higher than intervention group, 6- 8 hours after catheterization. Increasing in pain level leads to increased blood pressure, heart rate and respiratory rate. ${ }^{7,} 9,23$ This finding confirms the effect of catecholamine releasing and its positive impact on pain qualities such as inotropic, chronotropic and dermatropic role on heart rate, respiratory rate. So, the ANN can be considered as a model for prediction the pain value based on patient's vital signals.

This method can be used for reducing the pain and determining the best patient's position by using vital signals after the angiography.

In this study, the participants were randomly placed in the one of different positions, supine and in bed by angle $15^{\circ}, 30^{\circ}, 45^{\circ}$ and $60^{\circ}$ upward. All patients after the 
procedure reminded in a fixed position of predetermined Randomization till to the end.

\section{Limitations}

A limitation of this study is that data base for learning the ANN were obtained from one patient's position while vital signs must be evaluated in different positions for each person and the best position determine by ANN. We will be able to develop this work in future study and apply ANN for finding a minimum pain in different condition.

Moreover, increasing the trained dataset with more patients will be able to develop a good model to predict the pain. Also using Dynamic neural network and other pattern recognitions will be able to make better results.

\section{Conclusion}

A new approach is developed based on the ANN, in order to estimate the pain value based on vital signals after angiography. The pain which obtained from the proposed method is closely in agreement with the results achieved by using the SRN within less than $11 \%$ tolerance. So, this method can be suggested for reliving the pain and determining the best patient's position after the angiography procedure.

\section{Acknowledgments}

The authors would like to thank all patients who have participated in this study. We would also like to thank the directors of Imam Ali and Imam Reza hospitals, nurses of cardiac catheterization, and post angiography wards of Imam Ali Hospital, and CCU wards of Imam Reza Hospital in Bojnurd. The proposal of study was accepted by ethic committee of North Khorasan University of Medical Sciences.

\section{Conflict of interest}

The authors declared no conflict of interest.

\section{Authors' contribution}

MAYH: concept, manuscript writing

MY: data analysis, manuscript writing

ZA: Data collection

PS, ZF: manuscript writing,

AG: concept, data collection, manuscript writing

\section{References}

1. Farmanbar $R$, Afshar Mohammadiyan $M$, Moghaddamniya MT, kazemnejad E, Salari A. The effect of position change and bed-rest duration after coronary angiography on vascular complications. Iranian J Crit Care Nurs. 2012;4(4):177-182. [FreeFullText]

2. Kalyani MN, Jamshidi N. Risk factors of Iranian patients with three vessels disease candidate for coronary artery bypass graft surgery (CABG). Pak J Med Sci. 2010;26(4):837-841. [FreeFullText]

3. Rezaei-Adaryani M, Ahmadi F, Mohamadi E, AsghariJafarabadi $M$. The effect of three positioning methods on patient outcomes after cardiac catheterization. J Adv Nurs. 2009 Feb;65(2):417-24. [PubMed] DOI: 10.1111/j.1365-2648.2008.04889.x

4. Lundén MH, Bengtson A, Lundgren SM. Hours during and after coronary intervention and angiography. Clin Nurs Res. 2006 Nov;15(4):274-89. [PubMed] DOI: $\underline{10.1177 / 1054773806291855}$

5. Smeltzer SC. Chronic IIIness and Disability. In: Smeltzer SC, Bare B, Hinkle JL, Cheever KH. Brunner \& Suddarth $S$, Text book of Medical- Surgical Nursing 12th ed. Lippincott: Williams \& Wilkins; 2010. p.217.

6. Younesi Heravi M, Akbari H, Yaghubi M. Design and construction of an optical system for monitoring the blood supply to tissues under pressure. JNKUMS. 2012;3(4):101-110. [FreeFullText]

7. Bruehl S, Chung OY, Ward P, Johnson B, McCubbin JA. The relationship between resting blood pressure and acute pain sensitivity in healthy normotensives and chronic back pain sufferers: the effects of opioid blockade. Pain. 2002 Nov;100(1-2):191-201. [PubMed] DOI: 10.1016/s0304-3959(02)00295-6

8. al'Absi M, Petersen KL. Blood pressure but not cortisol mediates stress effects on subsequent pain perception in healthy men and women. Pain. 2003 Dec;106(3):28595. [PubMed] DOI: 10.1016/s0304-3959(03)00300-2

9. Lu Q, Zeltzer LK, Tsao JC, Kim SC, Turk N, Naliboff BD. Heart rate mediation of sex differences in pain tolerance in children. Pain. 2005 Nov;118(1-2):185-93. [PubMed] DOI: $10.1016 /$ j.pain.2005.08.008

10. Drummond PD. The effect of pain on changes in heart rate during the Valsalva manoeuvre. Clin Auton Res. 2003 Oct;13(5):316-20. [PubMed] DOI: 10.1007/s10286-003-0127-1

11. Smeltzer SC. Chronic Illness and Disability. In: Smeltzer SC, Bare B, Hinkle JL, Cheever KH. Brunner \& Suddarth $S$ Text book of Medical- Surgical Nursing.12th ed. Lippincott Williams\& Wilkins; 2010. p.145.

12. Qeethara Kadhim AS, Artificial Neural Networks in Medical Diagnosis, IJCSI. 2010;8(2):150-154. [FreeFullText]

13. Dybowsk R, Gant V. Clinical Applications of Artificial Neural Networks. Cambridge University Press; 2007.

14. Christopher BM. Neural networks for pattern recognition. Oxford University, USA; 1995. 
15. Haykin S. Neural networks, a comprehensive foundation. Prentice Hall International Editions; 1999.

16. Chair SY, Taylor-Piliae RE, Lam G, Chan S. Effect of positioning on back pain after coronary angiography. $J$ Adv Nurs. 2003 Jun;42(5):470-8. [PubMed] DOI: 10.1046/j.1365-2648.2003.02646.x

17. Demuth $H$, Beale $M$, Hagan M. Neural network toolbox 5 user's guide. The Mathworks: Natick; 2007.

18. Kozma R, Sakuma M, Yokoyama Y, Kitamura M. On the accuracy of mapping by neural networks trained by backpropagation with forgetting. Neurocomp. 1996;13(2):295-311. DOI: 10.1016/09252312(95)00094-1

19. Kasabov NK. Foundations of neural networks fuzzy systems and knowledge engineering. MIT: Cambridge; 1998.
20. Chair SY, Thompson DR, Li SK. The effect of ambulation after cardiac catheterization on patient outcomes. J Clin Nurs. 2007 Jan;16(1):212-4. [PubMed] DOI: 10.1111/j.1365-2702.2006.01599.x

21. Benson $\mathrm{G}$. Changing patients' position in bed after nonemergency coronary angiography reduced back pain. Evid Based Nurs. 2004 Jan;7(1):19. [PubMed] DOI: 10.1136/ebn.7.1.19

22. Coyne C, Baier W, Perra B, Sherer BK. Controlled trial of backrest elevation after coronary angiography. Am J Crit Care. 1994 Jul;3(4):282-8. [PubMed]

23. Pickering TG. Effects of stress and behavioral interventions in hypertension. Pain and blood pressure. $\mathrm{J}$ Clin Hypertens (Greenwich). 2003 Sep-Oct;5(5):35961. [PubMed] DOI: $10.1111 /$ j.1524-6175.2003.02830.x 